\title{
Pin\&Play: Bringing Power and Networking to Wall-Mounted Appliances
}

\author{
Kristof Van Laerhoven ${ }^{\unlhd}$, Nicolas Villar ${ }^{\unlhd}$, Maria Håkansson ${ }^{\dagger}$, and Hans-Werner Gellersen ${ }^{\unlhd}$ \\ ${ }^{\triangle}$ Ubiquitous Computing Group, \\ Lancaster University, \\ LA1 4YR Lancaster, United Kingdom \\ ${ }^{\dagger}$ Future Applications Lab, \\ Viktoria Institute, \\ Goteborg, Sweden
}

\{kristof,nvillar,hwg\}@comp.lancs.ac.uk,mariah@viktoria.se

\begin{abstract}
The classic obstacles in the digital augmentation of everyday objects and appliances are usually situated around batteries, communication, size, and weight. Wired communication is often not possible, changing or charging batteries is generally considered a nuisance, and augmenting an appliance is usually not worth it if it means increasing its size or weight. For the subclass of devices an objects that are typically attached to surfaces such as walls and boards we consider surface augmentation to address this problem. We present several implementations where low-cost wallpaper, consisting of insulated conductive layers, provides both power and a networking bus to common devices like pins on a notice board, wall switches, lights, and thermostats. They can be placed and replaced at will in any orientation, while the attachment to the wall supplies power and networking as a bonus. Moreover, our prototypes show that this doesn't affect familiarity of the objects, which makes interaction with them a breeze.
\end{abstract}

\section{Introduction}

Appliances that are large in size because of their functionality, like refrigerators, TV sets, dishwashers, vending machines, etc. usually remain on the same spot for long periods. It is both easy and worthwhile to provide a wall socket for them. A lot of objects that we use in our daily lives are, however, used in different locations. Many of those are furthermore small in size, and their form-factor doesn't allow them to be plugged in a wall socket or have a network connector.

Placing electronics in these objects or augmenting them with networking capabilities therefore becomes difficult. Build-in batteries might provide a solution in some cases, but this often means an unacceptable increase in size and weight of the appliance in the worst case, or a tedious changing or recharging of batteries in the best case. Socalled cradles provide a means of networking devices like mobile phones, personal digital assistants (PDAs), digital cameras, or laptop computers, but these are different for each device and need to be thoroughly distributed.

This paper presents current research that focuses on a certain class of objects that is typically attached to the wall. These objects could be devices we are already familiar with, such as wall-mounted clocks, wall switches, wall lights, posters, thermometers, pushpins, etc. or they could be completely new. A visual characteristic of these objects is that they have a pinconnector. The fastening to the wall not only provides placement and physical support, but additionally power and network connection. Introducing these devices to a power and networking source is therefore as straightforward as pinning them to the wall, hence the project's name: Pin\&Play.

\section{Characteristics of Pin\&Play}

Pin\&Play is based on the vision that walls and other common surfaces can be used as ad hoc bus network for objects that become attached to them [7]. This is a vision that requires a novel network composition, and that is concerned with qualities not typically considered in networking, e.g. calm facilitation of everyday environment and exploitation of design affordances for ease of use.

\subsection{Pin\&Play Components}

Surface. The purpose of the Pin\&Play physical network medium is to provide both network connectivity and power to attached objects. It is based on conductive sheets rather than on wires as the objective is to facilitate entire surfaces as two-dimensional network. As solid sheets would leave holes when pushpin connectors are 
inserted and later removed, fiber sheets are used instead. Pin\&Play Surfaces are composed of multiple layers of such sheets embedded in common surfaces. An anticipated challenge with the use of sheets rather than wires is that the resistance and the capacitance can be expected to increase faster over short distance, as surfaces get larger. However, a range of conductive materials are available that are optimized for low resistance. In general, the surface design is aimed at simplicity and low-cost, for example avoiding subdivision into tiles, to hold up our vision of deployment in everyday environments. Deployment of the surface material could for instance be envisioned in the form of smart lining under standard wall paper in the home, to enable entire walls as shared medium for objects that are attached to them.

Connectors. The design of Pin\&Play connectors is aimed to support two very different functionalities in a single mechanism. First, they obviously have to support physical connection of Pin\&Play network nodes to the surfaces (they would be the plugs if the network were not socket-less). Secondly, they should support attachment of objects based on existing and familiar practices. The connector design is therefore based on pushpins that can be stuck into Surfaces, and that can be removed as easily, thus employing a truly ubiquitous device that is commonplace in home and work environments. The connector design is inspired by related work in the Pushpin Computing project [2]. However our connectors are aimed at flexible augmentation of objects and hence conceived as adapter rather than built-in physical interface.

Pin\&Play Objects. The very idea of Pin\&Play is to provide networking to objects that are commonly attached to surfaces, rather than to conventional computing devices. In general, we consider two different types of object. First, we envision that any kind of object that people would attach to vertical surfaces can be "upgraded" to a networked object while also retaining its original appearance, purpose and use. This would apply for example for picture frames, artwork, wall calendars, clocks, light controls, and so on. Secondly, we envision objects that succeed today's mundane and ubiquitous connectors and fasteners, for example "Smart Pushpins" that can be used to attach notes to boards but that in addition provide new functionality on the basis of being digital and networked. Obviously, both types of objects require unobtrusive embedding of computation and network interface. In this context it has to be noted that Pin\&Play objects do not require their own power supply unless they are required to be "on" in detached mode.
Pin\&Play Network. Objects become powered up when they are attached to a surface. It is the task of the network to discover newly attached objects and to maintain network state. The network further has to provide the communication protocols for the connected nodes. A primary optimization target for the network is to support large-scale surfaces, high density of nodes, and ad hoc integration of previously unknown objects, while bandwidth is of lesser concern.

\subsection{Properties of Pin\&Play}

The design of Pin\&Play differs substantially from other conventional computer networks and other networks proposed specifically for ubiquitous computing.

Networking Properties. Pin\&Play addresses a design space between wired and wireless technologies. On an imaginary scale of ubiquity of network access, it goes beyond one-dimensional wired structures in providing network access across 2D surfaces, while of course not going as far as offering connection throughout 3D volumes. However, we consider it likely that overall higher density of nodes per room can be achieved if the enclosing walls were networked, in comparison to the state of the art in wireless technologies. A main advantage of Pin\&Play over wireless technologies is that it provides power to connected objects, and thus supports the integration of objects that have no batteries or other power supply. The approach is very similar to that of a PDA-cradle or a laptop docking station, but with minimal constraints concerning where and how to connect the object, and with more direct interconnection possibilities. Finally it has to be noted that surface-based networks have also been investigated in the Networked Surface project, however with focus on dedicated workplace surfaces with relatively complex internal structure [3].

Use-Related Properties. We already stressed that the Pin\&Play concept is firmly built on common structures. It addresses important user values such as familiarity of the concepts used, better observability of network configuration, and straightforward control in the sense of minimal-effort attachment and detachment of objects. Pushpin-like connectors provide a strong affordance, and the user act of connecting an object to the network becomes embedded in the act of attaching it to the wall or other surface. Another important property is the free placement of objects on a Pin\&Play Surface. People use surfaces for meaningful spatial arrangement of objects and therefore it is valuable that surface augmentation does not constrain such use. 
Deployment Vision. Pin\&Play is at this stage of our investigation a highly speculative technology, however its design is clearly targeted at real deployment in everyday environments. The components underlying Pin\&Play, in particular the layered conductive fiber sheets and the pushpin-like connectors, require careful design but do not involve sophisticated or expensive technology. If a satisfactory design is achieved, production at low-cost would certainly be realistic.

\subsection{Actual Implementation of Pin\&Play}

The wall as a network. The physical augmentation of a surface to a network bus was achieved by using two conductive layers separated by an insulating material such as cork or rubber. The conductive fiber sheets [4], which are traditionally manufactured for shielding applications, are not only straightforward to apply; they also leave no holes once the pins have been removed which ensures it can be used longer than solid conductive sheets. The fabric is usually silver $(\mathrm{Ag})$ or nickel-copper $(\mathrm{Ni}-\mathrm{Cu})$ plated nylon and has a typical thickness of 0.1 millimeters (0.005 inches).

To facilitate implementation of the network protocol, the Dallas MicroLAN [1] was chosen as the base communication layer, as it is an inexpensive and flexible networking standard, only requiring a single wire plus a ground reference. A large variety of network components is available and range in application from simple identification devices, read/write memories, to sensors and switches.

MicroLAN makes it is perfectly possible to build huge networks with many components that can be dynamically added or removed, it supports interrupting and requires a pull-up voltage between 2.8 and 6 Volts. This scalability and flexibility comes with a price though: the maximum communication bandwidth is only 16300 bits per second. The structure of the MicroLAN is furthermore a masterslave architecture, where the master could be seen as just another pin, accessing both layers to provide the essential power and communication to the board. Although implementing the network controller embedded like this is not unimaginable, a MicroLAN to serial interface is used at the moment to let a regular PC control the network. The aim of the network master's software is to find out what pins are plugged in, what components they contain and what they expect from the network.

Pin connectors. Our current prototype shown on the left in figure 1 has two isolated pins to get into the front and back layer of the Pin\&Play surface, much like that of

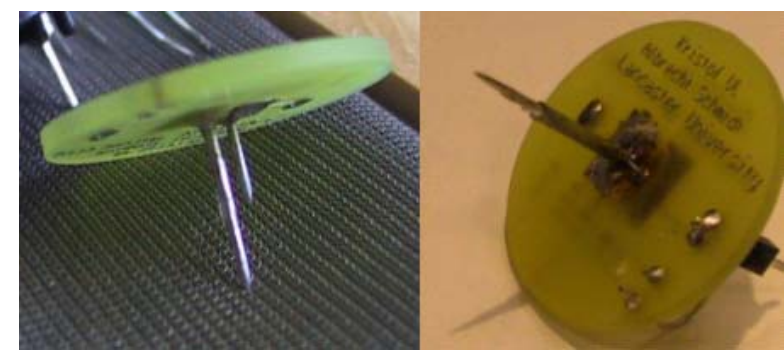

Figure 1. The two connectors. Left: the current twopin arrangement, right: the prototype with one pin.

the Pushpin Computing approach [2]. This connector design, with the larger pin partly covered with a transparent insulator has the disadvantage of being fixed in orientation once it has been plugged in, and being more vulnerable to short-circuits since the tips of both pins are more accessible. Additionally, the two-pin configuration is slightly harder to plug in, and alienates the Pin\&Play pin from traditional pins. It does however already illustrate the ease of pinning an object into the surface to provide it power and networking capabilities. As an alternative, we consider a single-pin design. Figure 1, right, shows the initial implementation, made from an industrial stainless steel nozzle (inner diameter: 0.58 $\mathrm{mms}$ ), as an outer cylinder containing a needle inside, both separated by insulating glue.

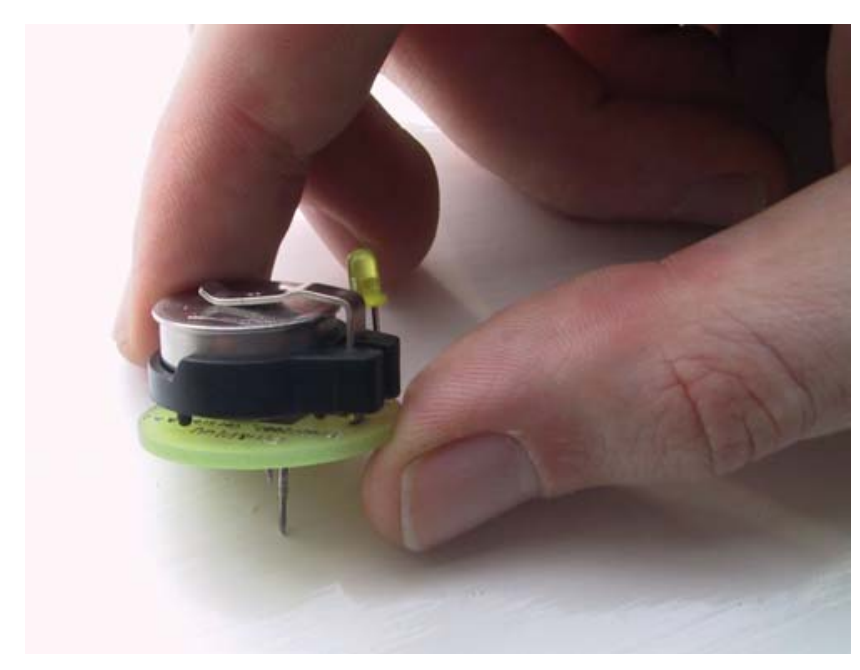

Figure 2. Close-up of an assembled pin. The current model has two isolated pins for accessing both layers. The one-wire switch is hidden below the i-Button's connector. 


\section{Examples}

After having discussed the bus system, consisting out of a physical surface with two conductive layers, insulated with rubber sheets, and a bus protocol, we now move on to the actual objects. We present two examples of pinaugmented objects and their networked application in this section.

\subsection{Notice Board Pins}

The first Pin\&Play objects that we prototyped are augmented versions of commonplace pushpins as found on noticeboards. Figure 2 shows our current prototype pin, which has at its heart a Time-in-a-Can iButton [6]. It is a self-sufficient component, containing its own battery (3V Lithium: with more than 10 years data retention!), oscillator $(32768 \mathrm{~Hz})$, memory (4096 bits), internal realtime calendar and clock (precision: 2 minutes/month), programmable alarms, and full MicroLAN communications support.

The augmented pushpin furthermore contains a LED that can be switched by a $1-$ Wire MicroLAN-compatible

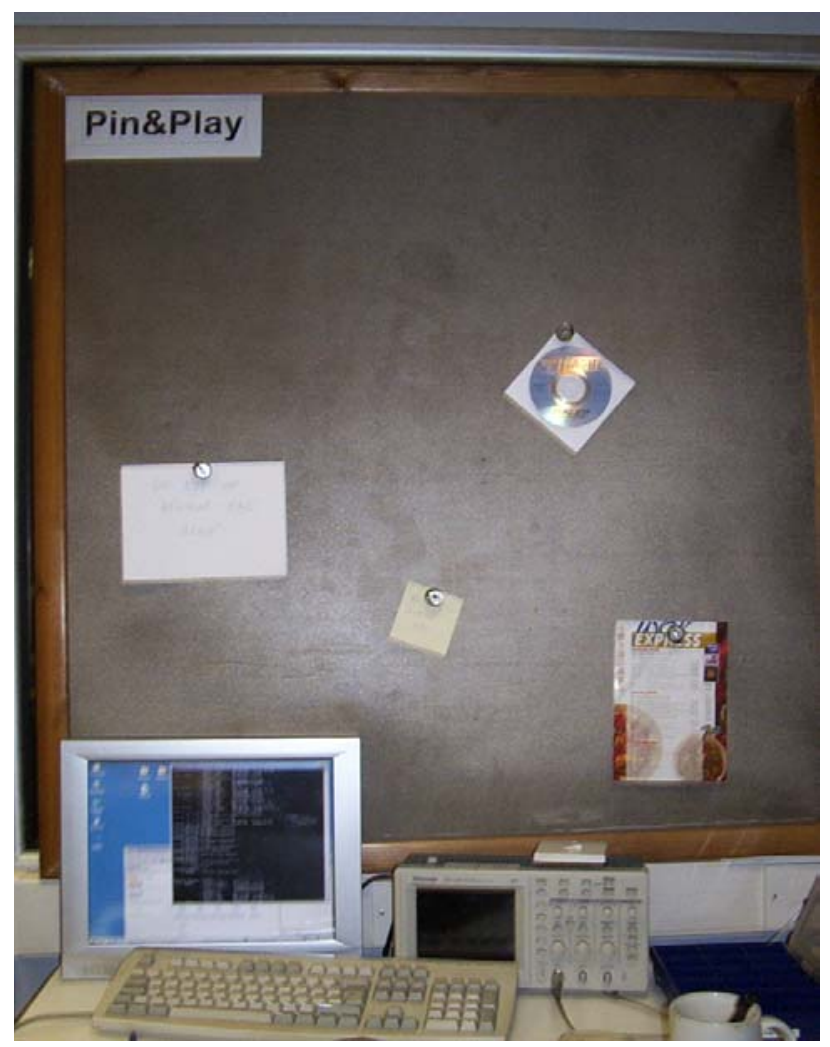

Figure 3. A typical setup, with the Pin\&Play board connected to a computer, acting as the network master. switch. Connecting a single pushpin hence introduces two devices into the network that are just physically bound in the same package. The Time-in-a-Can component has internal memory, where it has stored its partner's unique address, so both components can be bound together in virtual space as well.

The pin is, in contrast to other miniature devices from similar research projects, reasonably cheap and robust. The total sum of required components for one pin is about 8 US dollars, using the current retail prices. Additionally, the rigid structure of the main component (i.e. an iButton, embodied in stainless steel) ensures reliability and a long

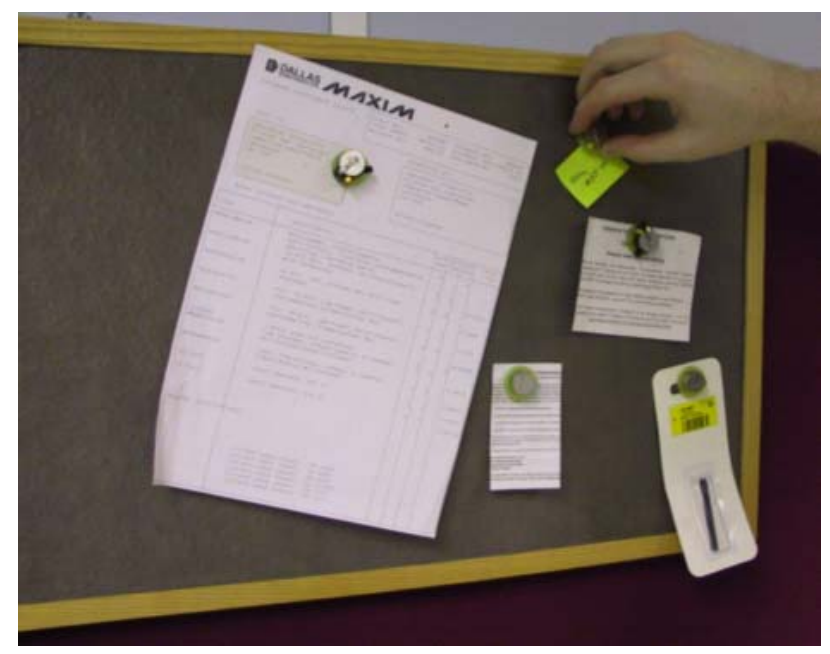

Figure 4. The Pin\&Play pins are used as normal pins, the Pin\&Play surface provides them with power and networking. The pins' batteries allow them to be moved from one board to another without losing any information.

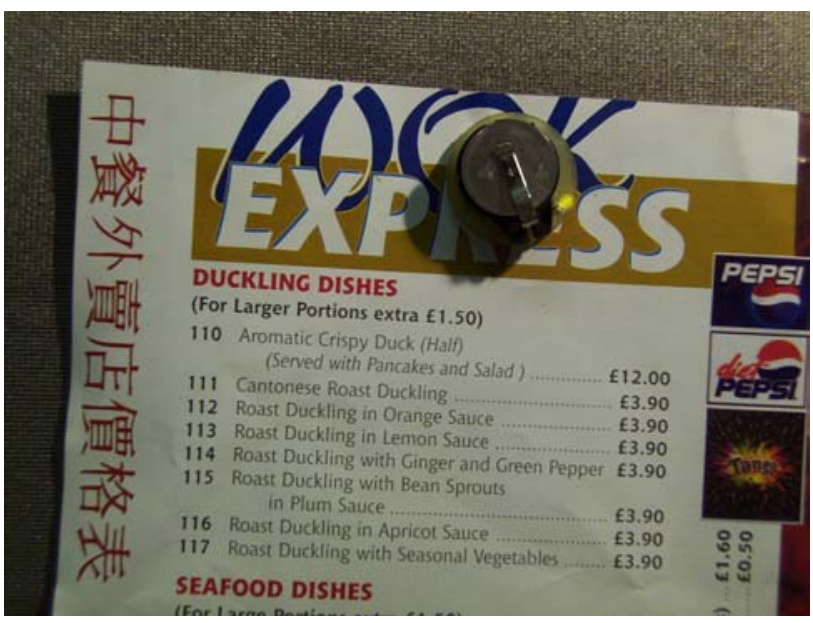

Figure 5. The pin's internal clock and calendar allow the pin to notify the user(s) when its document becomes invalid by lighting up its LED. 
life time despite it being a prototype. The tiny dimensions of especially the Time-in-a-Can iButton $(1.6 \mathrm{~cm}$ diameter, $0.6 \mathrm{~cm}$ height) produce a total size of a Pin\&Play pin that is close to its traditional equivalent.

Many applications are obvious with this hardware platform. The first one that we implemented involved assigning an expiration date for the document that is attached to the wall or notice board. Whenever the internal alarm of a pin goes off, it can - via the network master - switch its LED to notify it needs to be removed.

The alarm pin might be a solution to 'overcrowded notice boards' in public spaces where nobody checks the validity of the documents. To avoid confusion, priority values can be set to each document as well, so that only one pin (the one with the highest priority) will light up at a time.

The highlighting of a pin can of course have other meanings, for instance a document becoming relevant, or documents that are of interest to the user in front of the notice board.

\subsection{Dynamic Wall Switches and Lights}

To illustrate that any wall-mounted object can be augmented in the same fashion as in the previous example, we focused our attention to one of the most common objects found on a wall: wall switches that control the lights in a room. Instead of the electrician deciding for you where these switches belong, one can put the Pin\&Play three-layered surface as wallpaper on the wall and use an augmented switch.

Figure 6 shows the front and internals of a Pin\&Play enabled switch. It can report to the network master when the device is being switched, after which the appropriate light can be switched on or off. Developing more elaborate appliances such as dimmers or 'smarter' switches is very straightforward. Moreover, the network master can keep logs, automatically activate lights, or monitor light activity by just modifying the software.

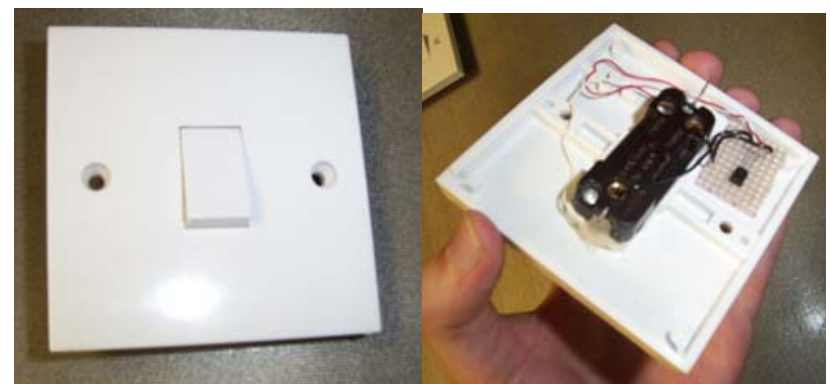

Figure 6. The front (left) and internals (right) of a Pin\&Play wall switch.

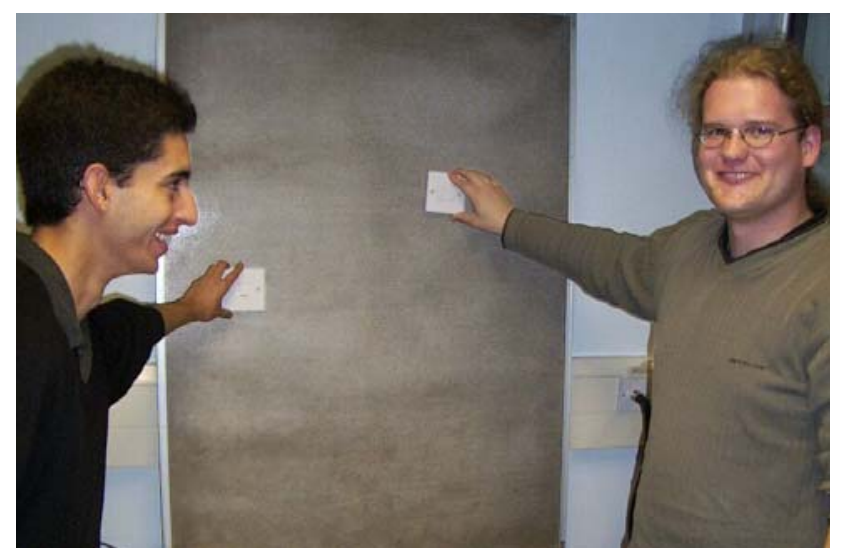

Figure 7. Placement of the wall switch is a matter of the user's preference, with the Pin\&Play switch.

The user will benefit from the dynamic placement of the switch: if the user desires the wall switch to be located in a different place or orientation, all that is required is pulling it out of the wallpaper and re-attaching it where preferred. Spotlights or wall-mounted lights can be attached and re-attached in the same fashion.

The insulating material used in this implementation is a flexible rubber sheet material with an adhesive side, to make it easier to glue the conductive fabric on. One could really imagine this process to be automation-friendly, enabling the production of flexible, layered wallpaper, which can be installed at low cost in both office and home environments.

\section{Conclusions}

This paper introduced the concepts of the Pin\&Play project, which aims at developing a highly integrated bus network in both office and domestic environments. By means of pin adaptors and a surface with layers of conductive sheets, ordinary devices can be wall-mounted to gain power and networking capabilities, with the freedom of being plugged in in any place or orientation.

The use of off-the-shelf components and the MicroLAN network protocol result in robust and small prototypes that are cheap, easy to (re)produce and yet more than powerful enough for the applications we envision. The network can handle many of devices in a small (though two dimensional) space, which is especially attractive in the augmentation of small and mobile appliances. 
We described some early prototyping and implementation of augmented pins and wall-mounted switches and lights as an indication of typical examples for the Pin\&Play concept. These examples serve as proof of concept for the Pin\&Play idea. Moreover they highlight that this technology has versatile uses and is by no means restricted to pinboard-based applications.

Future work in the project is concerned with assessing scalability of the network to test our hypothesis that we can achieve very high density networks with hundred or more objects connected over a single wall surface. Further work will also encompass the design and implementation of further applications. For this we will adopt a participatory design approach and engage users in the process to test our assumptions regarding the simplicity and ease of use of Pin\&Play.

\section{Acknowledgements}

The Pin\&Play assessment project is funded by the Commission of the European Union as part of the IST programme under contract IST-2001-37007. The first author also receives support for this research from the Engineering and Physical Sciences Research Council (EPSRC) in the United Kingdom, as part of the Equator Interdisciplinary Research Challenge.

\section{References}

1. "Dallas Reference Manual MicroLAN Starter Kit (DS9091K)" http://pdfserv.maximic.com/arpdf/DS9091K.pdf

2. J. Lifton, D. Seetharam, M. Broxton, J. Paradiso, "Pushpin Computing System Overview: a Platform for Distributed, Embedded, Ubiquitous Sensor Networks," in F. Mattern and M. Naghshineh (eds): Pervasive 2002, Proceedings of the Pervasive Computing Conference, Zurich Switzerland, 26-28 August 2002, Springer Verlag, Berlin Heidelberg, pp. 139-151.

3. Scott, F. Hoffmann, G. Mapp, M. Addlesee, A. Hopper. Networked Surfaces: A New Concept in Mobile Networking. In Proc. of WMCSA 2000, December 2000, Monterey, California, IEEE Press.

4. Shieldex Conductive woven sheets: http://www.shieldex.com

5. Technical Brief: 1-Wire Net Design Guide: http://www.maxim-ic.com/1st pages/tb1.htm

6. “Time-in-a-Can", DS1994 4-Kbit Plus Time Memory iButton datasheet. http://pdfserv.maximic.com/arpdf/DS1992-DS1994.pdf

7. Van Laerhoven, K, Schmidt A, and Gellersen, H-W. "Pin\&Play: Networking Objects through Pins". In Proceedings of Ubicomp 2002, G. Boriello and L.E. Holmquist (Eds). Lecture Notes in Computer Science; Vol. 2498, ISBN 3-540-44267-7; Springer Verlag, Göteborg, Sweden. September 2002, pp. 219-228 